\title{
Qualidade de vida de pessoas vivendo com HIV atendidas em serviços públicos de saúde
}

\author{
Quality of life of people living with HIV treated in public health services
}

Calidad de vida de personas viviendo con VIH atendidas en servicios públicos de salud

\author{
Hellen Pollyanna Mantelo Cecilio'; Daniela Sousa Oliveira"'; Sergio Corrêa Marques"II, \\ Thémistoklis Apostolidis ${ }^{\prime V}$; Denize Cristina de Oliveira ${ }^{V}$
}

\begin{abstract}
RESUMO
Objetivo: descrever o perfil e avaliar a qualidade de vida de pessoas vivendo com HIV atendidas em serviços públicos de saúde. Métodos: estudo transversal, quantitativo com 281 pessoas vivendo com HIV atendidas em serviços públicos de saúde nos municípios do Rio de Janeiro e Niterói. Os dados foram coletados por meio de instrumento de dados socioeconômicos e clínicos e do WHOQOL-HIV bref, analisados por estatística descritiva, após aprovação do Comitê de Ética em Pesquisa. Resultados: a amostra foi composta majoritariamente por homens, renda de até dois salários mínimos e percepção positiva da saúde. A qualidade de vida foi positivamente avaliada na maioria das facetas, as maiores médias foram nos domínios: psicológico, relações sociais, espiritualidade, religião e crenças pessoais e a menor no domínio meio ambiente. Conclusão: o perfil das pessoas vivendo com HIV corrobora, os dados nacionais e a avaliação da qualidade de vida foi mais positiva do que em outros estudos. Descritores: Qualidade de vida; HIV; soropositividade para HIV; enfermagem.
\end{abstract}

\begin{abstract}
Objective: to describe the profile and evaluate the quality of life of people living with HIV treated in public health services. Methods: in this cross-sectional, quantitative study of 281 people living with HIV treated in public health services in the municipalities of Rio de Janeiro and Niteroi, data were collected using a socioeconomic and clinical instrument and the WHOQOL-HIV brief questionnaire, and analyzed by descriptive statistics, with research ethics committee approval. Results: the sample comprised mainly men, with income of up to two minimum wages and a positive perception of their health. Most facets of quality of life were positively assessed, with the highest averages in the psychological, social relations, spirituality, religion and personal beliefs domains, and the lowest, in the environmental domain. Conclusion: the profile of people living with HIV corroborates national data, and the assessment of quality of life was more positive than in other studies.
\end{abstract}

Descriptors: Quality of life; HIV; HIV seropositivity; nursing.

\section{RESUMEN}

Objetivo: describir el perfil y evaluar la calidad de vida de personas viviendo con VIH atendidas en servicios públicos de salud. Métodos: estudio transversal, cuantitativo, junto a 281 personas viviendo con $\mathrm{VIH}$, atendidas en servicios públicos de salud en los municipios de Río de Janeiro y Niterói. Se han recolectado los datos mediante un instrumento de datos socioeconómicos y clínicos y del WHOQOL-HIV bref, analizados por estadística descriptiva, después de la aprobación del Comité de Ética en Investigación. Resultados: la muestra se compuso mayoritariamente de hombres, con ingresos de hasta dos salarios mínimos y percepción positiva de la salud. Se evaluó positivamente la calidad de vida en la mayoría de las facetas, los promedios más altos en los dominios: psicológico, relaciones sociales, espiritualidad, religión y creencias personales y el más bajo en el dominio medio ambiente. Conclusión: el perfil de las personas que viven con VIH corrobora con los datos nacionales y la evaluación de la calidad de vida fue más positiva que en otros estudios.

Descriptores: Calidad de vida; VIH; seropositividad para VIH; enfermería.

\section{INTRODUÇÃO}

Dados do UNAIDS (The Joint United Nations Programme on HIV/AIDS) apontaram 36,7 milhões de pessoas vivendo com (HIV) Vírus da Imunodeficiência Humana em 2017 no mundo, incluindo adultos e crianças. Em 2016 foram 1,8 milhões de novos casos de infecção pelo HIV e um milhão de óbitos relacionados à Síndrome da Imunodeficiência Adquirida (AIDS) em todo o mundo; na América Latina, estima-se 1,8 milhões de pessoas vivendo com HIV, registrando 36.000 óbitos e 97.000 novas infecções. Ainda apresentam redução de 48\% nos óbitos entre 2005 e 2016 devido ao aumento do acesso a terapia antirretroviral (TARV) ${ }^{1}$.

'Enfermeira. Doutoranda em Enfermagem, Universidade do Estado do Rio de Janeiro, Brasil. E-mail: pollymantelo@gmail.com "Enfermeira. Doutoranda em Enfermagem, Professora Auxiliar. Universidade do Estado da Bahia, BA, Brasil. E-mail: oliverdany@gmail.com

I'Enfermeiro. Doutor. Professor Adjunto, Universidade do Estado do Rio de Janeiro, Rio de Janeiro, RJ, Brasil. E-mail: sergiocmarques@uol.com.br Iv Psicólogo. Doutor. Aix-Marseille Université, Laboratoire de Psychologie Sociale. Aix-en-Provence, France. E-mail: themistoklis.apostolidis@univ-amu.fr VEnfermeira. Doutora. Professora Titular. Universidade do Estado do Rio de Janeiro, Faculdade de Enfermagem. Rio de Janeiro, RJ, Brasil. E-mail: dcouerj@gmail.com

V'Agradecimento ao CNPq pelo financiamento - Processo 485797/2013-5. 
Atualmente, a epidemia brasileira concentra-se em populações-chave como gays, homens que fazem sexo com homens, travestis e transexuais, pessoas que usam drogas e profissionais do sexo. A faixa etária com maior concentração dos casos é de 25 a 39 anos de ambos os sexos, sendo 53,6\% em homens. Os dados apontam aumento nos casos em homens com idades entre 15 a 29 anos, 20 a 24 anos e 60 anos e mais e queda nos casos em homens com idades entre 35 a 39 anos e 40 a 44 anos $^{2}$.

No contexto atual, permanece como uma epidemia global que continua a colocar desafios em nível político e na vida cotidiana das pessoas vivendo com HIV, tais como: aumento do número de pessoas infectadas; pobreza, discriminação e estigma; acesso aos serviços de saúde; manutenção de vínculos empregatícios; relações com os profissionais de saúde; vivência da sexualidade; alterações corporais e percepção da autoimagem; dificuldades com o suporte social e relações familiares, que interferem na avaliação da qualidade de vida dessas pessoas ${ }^{3-5}$.

Compreendendo a qualidade de vida a partir de um conceito amplo, que incorpora a saúde física, psicológica, as relações sociais e com o meio e o nível de independência; este estudo teve por objetivo descrever o perfil e avaliar a qualidade de vida de pessoas vivendo com HIV atendidas em serviços públicos de saúde.

\section{REVISÃO DE LITERATURA}

$\mathrm{Na}$ análise da história evolutiva da epidemia do HIV percebe-se a qualidade de vida em dois momentos, um antes e outro após o advento da TARV, destacando a interface psicossocial frente à possibilidade de viver com uma condição crônica ${ }^{6}$. Após a TARV, a Organização Mundial da Saúde (OMS) incluiu a AIDS na categoria das condições crônicas, como doença tratável e clinicamente manejável, considerando os avanços sobre a história natural da infecção, a possibilidade de monitorar a progressão e os avanços nos medicamentos antirretrovirais ${ }^{3,7}$.

Embora haja inúmeras definições, para a OMS, qualidade de vida reflete a percepção dos indivíduos de que suas necessidades estão sendo satisfeitas ou, ainda, que lhes estão sendo negadas oportunidades de alcançar a felicidade e a autorrealização, com independência de seu estado de saúde físico ou das condições sociais e econômicas ${ }^{8}$. A partir dessa conceituação, foram desenvolvidos instrumentos para a avaliação da qualidade de vida em uma perspectiva transcultural, entre eles o WHOQOL-HIV bref, composto por facetas que avaliam os diferentes aspectos da qualidade de vida, distribuídos em seis domínios: físico, psicológico, nível de independência, relações sociais, meio ambiente e espiritualidade, religião e crenças pessoais ${ }^{9}$.

O domínio físico contém facetas que avaliam a qualidade de vida relacionada a dor e desconforto, sono e repouso, energia e fadiga; o psicológico avalia sentimentos positivos e negativos, memória, concentração, autoestima, imagem corporal e aparência; o nível de independência contém questões sobre mobilidade, atividades diárias, dependência de tratamento e medicamentos e capacidade para o trabalho; o domínio relações sociais avalia as relações sociais, apoio social e atividade sexual; o meio ambiente avalia a qualidade de vida em relação a segurança, moradia, recursos financeiros, cuidados de saúde, lazer e transporte; o domínio espiritualidade, religião e crenças pessoais avalia as formas de espiritualidade, praticadas ou não através de religiões e, para os que não são afiliados a religião ou dimensão espiritual, refere-se a crenças ou códigos de comportamento ${ }^{9}$.

\section{Metodologia}

Estudo transversal, com recorte quantitativo, adotando amostragem do tipo não probabilística, de conveniência, a partir de informações disponibilizadas pelos serviços de saúde do quantitativo de pessoas em acompanhamento. A amostra foi composta por 281 pessoas vivendo com HIV, sendo 101 de Niterói e 180 do município do Rio de Janeiro.

Os indivíduos foram convidados a participar conforme a presença nos serviços de saúde nos períodos estabelecidos para a coleta dos dados, até completar o número definido em cada município. Sempre que ocorreram recusas ou desistência, novos indivíduos foram convidados em substituição, até a totalização da amostra, sendo selecionados os primeiros a aceitarem participar voluntariamente do estudo.

Os critérios de inclusão dos participantes foram: ter sorologia positiva para o HIV; estar em acompanhamento no serviço selecionado; estar aguardando consulta, informações ou exames durante o período de coleta de dados; ter idade maior ou igual a 18 anos; estar em condições clínicas e psicológicas que viabilizassem a participação. Como critério de exclusão, os indivíduos que se declaravam analfabetos, com deficiência visual importante ou falta de condição clínica foram substituídos, devido à necessidade de leitura e interpretação para responder os questionários autoaplicados.

A coleta de dados foi realizada entre março e outubro de 2016 em um Serviço de Atendimento Especializado em HIV/AIDS em Niterói e em três Centros Municipais de Saúde no município do Rio de Janeiro. Os dados foram coletados através do instrumento de dados socioeconômicos e clínicos e do WHOQOL-HIV bref $f^{8,9}$.

Os dados foram analisados com o software SPSS ${ }^{\oplus}$. A primeira etapa da análise configurou-se na descrição das frequências e categorização das variáveis socioeconômicas e clínicas; a segunda etapa consistiu no cálculo dos escores 
dos seis domínios da qualidade de vida realizado por meio de médias simples, agrupando as questões correspondentes a cada domínio ${ }^{8}$. Para interpretação dos escores da qualidade de vida, utilizou-se a seguinte classificação: médias acima de 15 consideradas como avaliação superior da qualidade de vida, médias entre 10 e 14,99 consideradas avaliação intermediária e, médias menores que 10 como avaliação inferior ${ }^{10}$.

Foram respeitadas as exigências dispostas na Resolução no 466/12 do Conselho Nacional de Saúde do Brasil, que versa sobre pesquisas envolvendo seres humanos e obteve todas as aprovações pertinentes. $O$ Termo de Consentimento Livre e Esclarecido foi disponibilizado a todos os participantes, garantindo o sigilo e anonimato.

\section{Resultados e Discussão}

\section{Perfil do grupo estudado}

As características socioeconômicas e clínicas dos participantes estão apresentadas na Tabela 1. A amostra foi composta majoritariamente por homens $(68,7 \%)$, corroborando dados nacionais que indicam $65 \%$ dos casos em pessoas do sexo masculino ${ }^{11}$. A quase totalidade dos participantes encontra-se em faixa etária economicamente produtiva, sendo 43,8\% dos casos entre 18 e 38 anos e 48,7\% dos casos entre 39 e 59 anos, com média de 41,1 anos (desvio-padrão $\pm 12,7$ anos). Estudos demonstram que a maioria dos casos de infecção pelo HIV encontra-se nas faixas etárias de 15 a 39 anos $^{2}$, destacando a faixa etária de 30 a $39 \operatorname{anos}^{3,12-15}$.

Relacionado à escolaridade, $74,7 \%$ declararam educação básica, que engloba ensino fundamental ao médio ${ }^{16}$, e $25,3 \%$ educação superior. Conforme encontrado em outros estudos a maioria das pessoas vivendo com HIV possui baixa escolaridade, um estudo constatou apenas $6 \%$ com ensino superior ${ }^{17}$ e outro, $80,2 \%$ com até sete anos de estudo ${ }^{18}$. A situação marital apresentou distribuição semelhante entre os participantes que referiram viver com companheiro $(50,5 \%)$ ou sem companheiro $(49,5 \%)$. Outros estudos ${ }^{3,19}$ também indicaram a maioria com companheiro, demonstrando que a situação de soropositividade não se apresenta como impeditiva de relações afetivas duradouras, embora indica a dificuldade de manutenção de parceiro fixo e o medo de compartilhar o diagnóstico diante da exposição a situações de preconceito e discriminação.

Relacionado à situação de emprego, a maioria (62,3\%) declarou-se empregada e 17,8\% desempregada. Essa taxa de desemprego provavelmente está mais relacionada com a situação do país no momento da coleta de dados, caracterizada por uma crise econômica, com altas taxas de desemprego, inflação elevada e descontentamento com a política $^{20}$, do que com a situação de soropositividade. Estudo ${ }^{21}$ com pessoas vivendo com HIV apresentou $12,7 \%$ desempregados e 9,2\% aposentados; já outro estudo ${ }^{15}$ revelou $15,9 \%$ de pessoas desempregadas, $24,8 \%$ aposentadas e $23,6 \%$ trabalhando.

Quanto à renda, mesmo considerando que a escolha das unidades de saúde tenha sido intencional para garantir a variabilidade socioeconômica da amostra, o percentual de pessoas com renda de até dois salários mínimos é maior. Tal situação corrobora com dados nacionais e internacionais que conferem à pobreza uma importante contribuição para o aumento da vulnerabilidade para a infecção por HIV². Ainda, a baixa renda encontrada confirma os dados do interior do estado do Rio de Janeiro, em que $37,4 \%$ das pessoas vivem com até três salários mínimos em Macaé ${ }^{3}$ e metade dos entrevistados vive com até dois salários mínimos em Rio das Ostras ${ }^{13}$, bem como os entrevistados na Paraíba, onde a maioria vive com até dois salários mínimos ${ }^{15}$.

Sobre a orientação religiosa, quando comparado com as demais opções, o grupo de católicos representou 31,4\%, portanto, minoritário; todavia, quando comparado com o grupo de evangélicos, sem religião ou outras religiões apresentou-se majoritário. Os dados deste estudo corroboram com os resultados encontrados em estudos realizados em Fortaleza/CE ${ }^{12}$ e em João Pessoa/PB ${ }^{15}$ que apontaram maioria católica; porém contesta a afirmativa de que o estado do Rio de Janeiro possui o menor quantitativo de católicos do país e ainda, contraria os dados nacionais que apontam tendência à diminuição de católicos, cujos percentuais variaram de 63,8\% para 50\%, de 2010 para 2016, enquanto os evangélicos aumentaram de $24 \%$ para $29 \%$, no mesmo período ${ }^{23}$.

Relacionado ao modo de exposição ao HIV, embora a relação homossexual tenha se apresentado como principal modo de exposição ao vírus $(43,1 \%)$, as relações heterossexuais também contribuíram para a transmissão sexual $(40,2 \%)$, como encontrado em estudo com homens no Nordeste ${ }^{12}$ e no Sul ${ }^{19}$. Ainda chama a atenção dados da África, em que $53,5 \%$ e $35,6 \%$ das pessoas entrevistadas não sabem informar como se infectaram ${ }^{24,25}$. Observa-se o baixo percentual de transmissão sanguínea, especialmente por drogas injetáveis $(0,7 \%)$, corroborando com dados do estado do Rio de Janeiro ${ }^{26}$. O UNAIDS considera que gays, homens que fazem sexo com homens, pessoas trans, profissionais do sexo, seus clientes e pessoas que usam drogas injetáveis são as principais populações-chave ${ }^{1}$, isto porque, muitas vezes essas populações são sujeitas a leis punitivas ou políticas estigmatizantes e têm mais probabilidade de exposição ao HIV. Dados nacionais apontam que os homens que fazem sexo com homens constituem a população-chave que corresponde pela maior parte de casos novos de infecção pelo HIV². 
TABELA 1: Caracterização das pessoas vivendo com HIV. Rio de Janeiro e Niterói Brasil, 2016. $(n=281)$

\begin{tabular}{|c|c|}
\hline Variáveis & $f(\%)$ \\
\hline \multicolumn{2}{|l|}{ Sexo } \\
\hline Masculino & $193(68,7)$ \\
\hline Feminino & $88(31,3)$ \\
\hline \multicolumn{2}{|l|}{ Faixa etária (em anos) } \\
\hline 18 a 38 & $123(43,8)$ \\
\hline 39 a 59 & $137(48,7)$ \\
\hline 60 e mais & $21(7,5)$ \\
\hline \multicolumn{2}{|l|}{ Escolaridade } \\
\hline Educação básica & $210(74,7)$ \\
\hline Educação superior & $71(25,3)$ \\
\hline \multicolumn{2}{|l|}{ Situação marital } \\
\hline Com companheiro & $142(50,5)$ \\
\hline Sem companheiro & $139(49,5)$ \\
\hline \multicolumn{2}{|l|}{ Situação de emprego } \\
\hline Com emprego & $175(62,3)$ \\
\hline Aposentado/outros & $56(19,9)$ \\
\hline Desempregado & $50(17,8)$ \\
\hline \multicolumn{2}{|l|}{ Renda em reais } \\
\hline Até R\$ 1734 (2 salários mínimos) & $159(56,6)$ \\
\hline $\mathrm{R} \$ 1735$ e mais & $122(43,4)$ \\
\hline \multicolumn{2}{|l|}{ Orientação religiosa } \\
\hline Católicos & $88(31,4)$ \\
\hline Não tem religião & $74(26,3)$ \\
\hline Evangélicos & $65(23,1)$ \\
\hline Outras religiões & $54(19,2)$ \\
\hline \multicolumn{2}{|l|}{ Exposição ao HIV } \\
\hline Relação homossexual & $121(43,1)$ \\
\hline Relação heterossexual & $113(40,2)$ \\
\hline Transmissão sanguínea & $45(16)$ \\
\hline Drogas injetáveis & $2(0,7)$ \\
\hline \multicolumn{2}{|l|}{ Orientação sexual } \\
\hline Heterossexual & $144(51,2)$ \\
\hline Homossexual/Bissexual & $137(48,8)$ \\
\hline \multicolumn{2}{|l|}{ Tempo de diagnóstico do HIV } \\
\hline Até 24 meses ( 2 anos) & $28(10)$ \\
\hline De 25 a 72 meses ( 2 anos e 1 mês a 6 anos) & $77(27,4)$ \\
\hline De 73 a 168 meses ( 6 anos e 1 mês a 14 anos) & $88(31,3)$ \\
\hline 169 meses e mais ( 14 anos e 1 mês e mais) & $88(31,3)$ \\
\hline \multicolumn{2}{|l|}{ Uso da TARV } \\
\hline Sim & $265(94,3)$ \\
\hline Não & $16(5,7)$ \\
\hline \multicolumn{2}{|l|}{ Alterações em relação a TARV $(n=265)$} \\
\hline Não tem ou teve alterações & $139(52,5)$ \\
\hline Tem ou teve alterações & $126(47,5)$ \\
\hline \multicolumn{2}{|l|}{ Estágio da infecção } \\
\hline Sem sintomas & $252(89,7)$ \\
\hline Com sintomas & $29(10,3)$ \\
\hline \multicolumn{2}{|l|}{ Considerar-se doente } \\
\hline Não & $234(83,3)$ \\
\hline Sim & $47(16,7)$ \\
\hline \multicolumn{2}{|l|}{ Percepção da saúde } \\
\hline Positiva & $233(82,9)$ \\
\hline Negativa & $48(17,1)$ \\
\hline
\end{tabular}


Tal proposição corrobora parcialmente com este estudo, já que a maior parte dos participantes se declarou do sexo masculino e $48,8 \%$ se declarou homossexual ou bissexual e $51,2 \%$ heterossexual. Estes resultados diferem do encontrado em Macaé ${ }^{3}$ e em João Pessoa ${ }^{15}$, cuja maioria se declarou heterossexual. Relacionado ao tempo de diagnóstico do HIV, mais da metade $(62,6 \%)$ relatou diagnóstico há mais de seis anos, semelhante ao encontrado em João Pessoa ${ }^{15}$ e diferente de Rio das Ostras ${ }^{13}$, em que $68 \%$ obtiveram o diagnóstico há menos de quatro anos.

Os resultados encontrados apontam maior utilização da TARV nesta amostra (94,3\%), visto que nos estudos realizados nos últimos anos no interior do estado, 77\% dos entrevistados utilizavam a terapia antirretroviral em Macaé ${ }^{3}$ e 74\% em Rio das Ostras ${ }^{13}$; ainda, em estudos recentes realizados na África, $80 \%$ dos entrevistados usavam TARV na Nigéria ${ }^{24}$ e 72,9\% em Burkina Faso ${ }^{25}$. A maior utilização da TARV certamente está relacionada com a recomendação da OMS para o início mais precoce do tratamento - contagem de T-CD4+ de 350 para 500 células por milímetros cúbicos a partir de 2013, diante das evidências dos benefícios clínicos ${ }^{27}$.

Relacionado às pessoas que fazem uso da TARV, pouco mais da metade $(52,5 \%)$ indicou que não tem ou teve alterações relacionadas à medicação, divergindo dos dados em que $80 \%$ das pessoas informaram ter essas alterações ${ }^{3}$, apontando a menor toxicidade dos ARV atualmente utilizados no Brasil. Os participantes informaram não se perceber doentes (83,3\%) e isso pode estar relacionado ao fato de que $89,7 \%$ afirmaram não apresentarem sintomas relacionados a infecção, mesmo vivendo com HIV. Entretanto, estudo realizado na Nigéria revelou que mesmo 26,9\% das pessoas estando em estágio sintomático e 53,3\% terem AIDS, 70,3\% não se consideraram doentes ${ }^{24}$.

A percepção positiva da saúde observada em 82,9\% dos participantes pode ser justificada por uma tendência a naturalizar o viver com o HIV, sendo percebida como adaptação e aceitação da soropositividade, conforme observado em outras pesquisas ${ }^{28,29}$. A boa percepção da saúde também foi encontrada em outros estudos, sendo positiva para $88,8 \%$ das pessoas entrevistadas na Nigéria ${ }^{24}$ e $63,7 \%$ em Burkina Faso ${ }^{25}$.

\section{Avaliação da Qualidade de Vida}

Em todas as facetas e domínios mensurados pelo WHOQOL-HIV bref, percebe-se uma avaliação positiva, visto que $78,9 \%$ avaliaram positivamente sua qualidade de vida e 68,9\% declararam satisfação com sua saúde. Essa posição global positiva teve frequência superior aos achados no interior do estado do Rio de Janeiro que as frequências foram $65,7 \%$

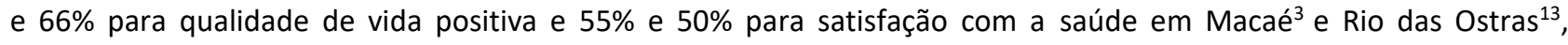
respectivamente. Tais apontamentos corroboram com o pensamento de alguns autores de que a possibilidade de cronicidade, com a introdução da TARV e, sobretudo, a convivência com o vírus por longo tempo sem manifestações da doença, propiciam uma visão mais positiva quanto à própria vida e a qualidade de vida ${ }^{30}$.

Os resultados da avaliação da qualidade de vida são apresentados na Tabela 2, refletindo o posicionamento das pessoas vivendo com HIV sobre os domínios da qualidade de vida.

TABELA 2: Distribuição dos escores entre os domínios do WHOQOL-HIV bref. Rio de Janeiro e Niterói - Brasil, 2016.

\begin{tabular}{lcccc}
\hline Domínios do WHOQOL-HIV bref & $\begin{array}{c}\text { Média } \\
\text { simples }\end{array}$ & $\mathbf{( \pm ) ~ d p ~}$ & $\begin{array}{c}\text { Média } \\
\text { multiplicada }\end{array}$ & $\mathbf{( \pm ) ~ d p ~}$ \\
\hline Autoavaliação da QV & 3,87 & 0,83 & 15,49 & 3,34 \\
Psicológico & 3,81 & 0,70 & 15,24 & 2,82 \\
Espiritualidade, religião e crenças pessoais & 3,75 & 0,93 & 14,99 & 3,74 \\
Relações Sociais & 3,74 & 0,83 & 14,96 & 3,34 \\
Físico & 3,68 & 0,86 & 14,72 & 3,45 \\
Nível de independência & 3,67 & 0,76 & 14,68 & 3,07 \\
Meio Ambiente & 3,37 & 0,64 & 13,50 & 2,57 \\
\hline
\end{tabular}

Para as pessoas vivendo com HIV neste estudo, a autoavaliação da qualidade de vida apresentou média de 15,49, indicando avaliação superior. O domínio psicológico apresentou a maior média $(15,24)$ e o menor desvio-padrão, indicando avaliação superior. Os demais domínios - Físico, Nível de Independência, Relações Sociais, Meio Ambiente e Espiritualidade, religião e crenças pessoais - aludem à avaliação intermediária ${ }^{10}$, com médias entre 10 e 14,99. As médias encontradas em todos os domínios neste estudo são superiores aos encontrados no interior do estado do Rio de Janeiro ${ }^{3,13}$

Os resultados das facetas correspondentes aos domínios da qualidade de vida estão na Tabela 3. 
TABELA 3: Distribuição das respostas dos participantes quanto às facetas do WHOQOL-HIV bref. Rio de Janeiro e Niterói - Brasil, 2016.

\begin{tabular}{|c|c|c|c|c|c|c|}
\hline \multicolumn{2}{|c|}{ Aspectos da Qualidade de Vida } & \multirow{2}{*}{$\begin{array}{c}\text { (1) } \mathrm{MN}^{(*)} \\
4\end{array}$} & \multirow{2}{*}{$\frac{\text { (2) Negativa }}{7}$} & \multirow{2}{*}{$\begin{array}{c}\text { (3) Médio } \\
48\end{array}$} & \multirow{2}{*}{$\begin{array}{c}\text { (4) Positiva } \\
144\end{array}$} & \multirow{2}{*}{$\frac{\text { (5) } \mathrm{MP}^{(* *)}}{77}$} \\
\hline Auto & Q1 (G1) (280) & & & & & \\
\hline \multirow[t]{3}{*}{ avaliação da QV } & Qualidade de vida & $1,4 \%$ & $2,5 \%$ & $17,1 \%$ & $51,4 \%$ & $27,6 \%$ \\
\hline & Q2 (G4) (280) & 11 & 24 & 52 & 126 & 67 \\
\hline & Satisfação com a saúde & $3,9 \%$ & $8,6 \%$ & $18,6 \%$ & $45 \%$ & $23,9 \%$ \\
\hline Domínio & Q6 (F4.1) (279) & 10 & 11 & 40 & 122 & 96 \\
\hline \multirow[t]{9}{*}{ Psicológico } & Aproveita a vida & $3,6 \%$ & $3,9 \%$ & $14,4 \%$ & $43,7 \%$ & $34,4 \%$ \\
\hline & Q11 (F5.3) (281) & 11 & 30 & 59 & 125 & 56 \\
\hline & Consegue concentrar & $3,9 \%$ & $10,7 \%$ & $21 \%$ & $44,5 \%$ & $19,9 \%$ \\
\hline & Q15 (F7.1) (280) & 8 & 22 & 56 & 91 & 103 \\
\hline & Aceita aparência física & $2,9 \%$ & $7,8 \%$ & $20 \%$ & $32,5 \%$ & $36,8 \%$ \\
\hline & Q24 (F6.3) (278) & 12 & 18 & 44 & 107 & 97 \\
\hline & Satisfeito consigo mesmo & $4,3 \%$ & $6,5 \%$ & $15,8 \%$ & $38,5 \%$ & $34,9 \%$ \\
\hline & Q31 (F8.1) (279) & 14 & 30 & 40 & 154 & 41 \\
\hline & Sentimentos negativos & $5 \%$ & $10,8 \%$ & $14,3 \%$ & $55,2 \%$ & $14,7 \%$ \\
\hline Domínio & Q7 (F24.2) (279) & 11 & 5 & 28 & 100 & 135 \\
\hline Espiritualidade & Vida tem sentido & $3,9 \%$ & $1,8 \%$ & $10 \%$ & $35,8 \%$ & $48,5 \%$ \\
\hline religião e crenças & $\mathrm{Q} 8$ (F52.2) (277) & 34 & 40 & 30 & 47 & 126 \\
\hline \multirow[t]{5}{*}{ pessoais } & Responsabilidade condição & $12,3 \%$ & $14,4 \%$ & $10,8 \%$ & $17 \%$ & $45,5 \%$ \\
\hline & Q9 (F53.2) (281) & 30 & 44 & 51 & 43 & 113 \\
\hline & Medo do futuro & $10,7 \%$ & $15,7 \%$ & $18,1 \%$ & $15,3 \%$ & $40,2 \%$ \\
\hline & Q10 (F54.1) (281) & 43 & 37 & 39 & 40 & 122 \\
\hline & Se preocupa com a morte & $15,3 \%$ & $13,2 \%$ & $13,9 \%$ & $14,2 \%$ & $43,4 \%$ \\
\hline Domínio Relações & Q27 (F14.4) (279) & 12 & 7 & 78 & 98 & 84 \\
\hline \multirow[t]{7}{*}{ Sociais } & Satisfeito apoio amigos & $4,3 \%$ & $2,5 \%$ & $28 \%$ & $35,1 \%$ & $30,1 \%$ \\
\hline & Q26 (F15.3) (276) & 24 & 23 & 70 & 99 & 60 \\
\hline & Satisfeito com vida sexual & $8,7 \%$ & $8,3 \%$ & $25,4 \%$ & $35,9 \%$ & $21,7 \%$ \\
\hline & Q25 (F13.3) (281) & 12 & 9 & 61 & 115 & 84 \\
\hline & Satisfeito relações pessoais & $4,3 \%$ & $3,2 \%$ & $21,7 \%$ & $40,9 \%$ & $29,9 \%$ \\
\hline & Q17 (F51.1) (279) & 10 & 23 & 58 & 105 & 83 \\
\hline & Aceito pelas pessoas & $3,7 \%$ & $8,2 \%$ & $20,8 \%$ & $37,6 \%$ & $29,7 \%$ \\
\hline Domínio & Q3 (F1.4) (281) & 8 & 32 & 49 & 44 & 148 \\
\hline \multirow[t]{7}{*}{ Físico } & Dor Física impede & $2,8 \%$ & $11,4 \%$ & $17,4 \%$ & $15,7 \%$ & $52,7 \%$ \\
\hline & Q4 (F50.1) (277) & 27 & 47 & 41 & 44 & 118 \\
\hline & Incomoda problema físico & $9,7 \%$ & $17 \%$ & $14,8 \%$ & $15,9 \%$ & $42,6 \%$ \\
\hline & Q14 (F2.1) (280) & 6 & 24 & 86 & 100 & 64 \\
\hline & Energia suficiente dia a dia & $2,1 \%$ & $8,6 \%$ & $30,7 \%$ & $35,7 \%$ & $22,9 \%$ \\
\hline & Q21 (F3.3) (281) & 14 & 53 & 71 & 95 & 48 \\
\hline & Satisfação com o sono & $5 \%$ & $18,9 \%$ & $25,3 \%$ & $33,7 \%$ & $17,1 \%$ \\
\hline Domínio & Q5 (F11.3) (279) & 42 & 86 & 58 & 62 & 31 \\
\hline Nível de & Precisa de tratamento & $15,1 \%$ & $30,8 \%$ & $20,8 \%$ & $22,2 \%$ & $11,1 \%$ \\
\hline \multirow[t]{6}{*}{ Independência } & Q22 (F10.3) (280) & 12 & 25 & 46 & 115 & 82 \\
\hline & Capacidade atividade diária & $4,3 \%$ & $8,9 \%$ & $16,4 \%$ & $41,1 \%$ & $29,3 \%$ \\
\hline & Q23 (F12.4) (278) & 13 & 18 & 52 & 120 & 75 \\
\hline & Capacidade para o trabalho & $4,7 \%$ & $6,5 \%$ & $18,6 \%$ & $43,2 \%$ & $27 \%$ \\
\hline & Q20 (F9.1) (280) & 4 & 9 & 33 & 86 & 148 \\
\hline & Capaz de se locomover & $1,4 \%$ & $3,2 \%$ & $11,8 \%$ & $30,7 \%$ & $52,9 \%$ \\
\hline Domínio & Q12 (F16.1) (281) & 19 & 21 & 72 & 135 & 34 \\
\hline \multirow[t]{15}{*}{ Meio Ambiente } & Seguro se sente vida diária & $6,8 \%$ & $7,5 \%$ & $25,6 \%$ & $48 \%$ & $12,1 \%$ \\
\hline & Q13 (F22.1) (281) & 16 & 26 & 105 & 106 & 28 \\
\hline & Saudável o ambiente físico & $5,7 \%$ & $9,3 \%$ & $37,4 \%$ & $37,6 \%$ & $10 \%$ \\
\hline & Q16 (F18.1) (280) & 31 & 67 & 116 & 32 & 34 \\
\hline & Dinheiro suficiente & $11,1 \%$ & $23,9 \%$ & $41,4 \%$ & $11,4 \%$ & $12,2 \%$ \\
\hline & Q18 (F20.1) (279) & 15 & 27 & 82 & 95 & 60 \\
\hline & Disponibilidade informações & $5,4 \%$ & $9,7 \%$ & $29,3 \%$ & $34,1 \%$ & $21,5 \%$ \\
\hline & Q19 (F21.1) (281) & 16 & 39 & 90 & 91 & 45 \\
\hline & Oportunidades de lazer & $5,7 \%$ & $13,9 \%$ & $32 \%$ & $32,4 \%$ & $16 \%$ \\
\hline & Q28 (F17.3) (280) & 13 & 27 & 56 & 103 & 81 \\
\hline & Satisfeito com a moradia & $4,6 \%$ & $9,6 \%$ & $20 \%$ & $36,9 \%$ & $28,9 \%$ \\
\hline & Q29 (F19.3) (280) & 18 & 33 & 52 & 106 & 71 \\
\hline & Acesso serviços de saúde & $6,4 \%$ & $11,7 \%$ & $18,6 \%$ & $37,9 \%$ & $25,4 \%$ \\
\hline & Q30 (F23.3) (281) & 42 & 64 & 71 & 74 & 30 \\
\hline & Satisfeito com o transporte & $14,9 \%$ & $22,8 \%$ & $25,3 \%$ & $26,3 \%$ & $10,7 \%$ \\
\hline
\end{tabular}


O domínio psicológico obteve a maior média $(4,3)$ entre os domínios e o menor desvio-padrão $(0,981)$, corroborando ao encontrado em Teresina/ $/ \mathrm{PI}^{14}$. Esse achado indica avaliação superior da qualidade de vida para as pessoas vivendo com HIV, análogo ao encontrado na Nigéria ${ }^{24}$ e em São José/SC ${ }^{31}$. Em contrapartida, difere dos resultados em Macaé ${ }^{3}$, Rio das Ostras ${ }^{13}$, Mossoró/RN ${ }^{32}$, África do Sul ${ }^{33}$ e Camboja ${ }^{34}$, classificados como intermediários.

Esse domínio contém cinco facetas e a primeira corresponde ao quanto o indivíduo aproveita a vida, sendo que $78,1 \%$ dos participantes responderam com uma avaliação muito positiva. Ressalta-se que no interior do estado $21,1 \%$ das pessoas referiram aproveitar muito pouco ou nada da $v_{i d a}{ }^{13}$, uma porcentagem grande quando comparada com este estudo, em que apenas 7,5\% consideraram não aproveitar a vida. A segunda faceta questiona sobre a capacidade de concentração e $64,4 \%$ perceberam-se capazes de se concentrar. Ainda, as facetas relacionadas à aceitação da aparência física e à satisfação consigo mesmo, obtiveram, respectivamente, $69,3 \%$ e $73,4 \%$ de respostas positivas. $A$ faceta relacionada aos sentimentos negativos revelou que $69,9 \%$ dos participantes não apresentam tais sentimentos com frequência. Quando comparado ao interior do estado, 59\% em Rio das Ostras ${ }^{13}$ referiram não ter sentimentos negativos frequentemente, todavia, em Macaé $^{3}, 71 \%$ das pessoas referiram frequentemente esses sentimentos negativos.

O domínio espiritualidade, religião e crenças pessoais contém quatro facetas e apresentou valor médio limítrofe entre intermediário e superior, sendo a segunda maior média entre os domínios. A classificação como intermediário corrobora com os achados em Macaé ${ }^{3}$, em Rio das Ostras ${ }^{13}$, na África do Sul ${ }^{33}$ e no Camboja ${ }^{34}$. Os dados na Nigéria ${ }^{24}$ e São José ${ }^{31}$ obtiveram avaliação superior e, em Mossoró ${ }^{32}$ teve média abaixo de dez, sugerindo avaliação inferior da qualidade de vida. A primeira faceta avaliou o quanto a vida tem sentido, sendo positiva para $84,2 \%$. A segunda faceta questionou a responsabilização atribuída pela condição de viver com HIV, revelando não incomodar $62,5 \%$ dos participantes. A terceira questionou o medo do futuro, que revelou não ser problema para 55,5\% das pessoas e a última, a preocupação com a morte, apontando que $57,6 \%$ das pessoas não tem preocupação nesse sentido.

O domínio relações sociais contém quatro facetas e apresentou a terceira maior média, sugerindo avaliação intermediária, porém, muito próximo do limite para a avaliação superior. Os dados na África do Sul ${ }^{33}$ e na $\mathrm{Nigéria}^{24}$ apontaram avaliação superior da qualidade de vida e os estudos em Macaé ${ }^{3}$, Rio das Ostras ${ }^{13}$, São José ${ }^{31}$ e Camboja ${ }^{34}$ avaliação intermediária. Em Fortaleza ${ }^{12}$ obteve a maior média diferente do relatado em São Paulo/SP ${ }^{10}$, revelando que as pessoas vivendo com HIV possuem melhor qualidade de vida nos domínios físico e psicológico do que as demais e pior no domínio relações sociais, o que pode refletir situações de estigma, preconceito e discriminação, o que parece não se observar neste estudo. A primeira faceta avalia o apoio recebido pelos amigos, que foi percebido como positivo por $65,2 \%$ das pessoas. A segunda questiona sobre a satisfação com a vida sexual, apontando satisfação para $57,6 \%$ dos participantes, semelhante ao encontrado em Rio das Ostras ${ }^{13}$ que $51 \%$ estavam satisfeitos e contrário ao encontrado em Macaé ${ }^{3}$, com insatisfação para $67,9 \%$. A terceira e quarta facetas avaliam, respectivamente, a satisfação com as relações pessoais e a aceitação pelas pessoas que conhece, sendo avaliada positivamente por 70,8\% e 67,3\% das pessoas vivendo com HIV.

O domínio físico contém quatro facetas e teve avaliação intermediária, semelhante aos achados na África do Sul ${ }^{33}$, Camboja ${ }^{34}$; e no Brasil, em Fortaleza ${ }^{12}$ e Mossoró ${ }^{32}$. Já os resultados em São José ${ }^{31}$ e na Nigéria ${ }^{24}$ apontaram escores sugestivos de avaliação superior. A primeira faceta investiga a presença de dor, que pode acompanhar alguns quadros evolutivos da doença e interferir na qualidade de vida, entretanto, a maioria $(68,4 \%)$ relatou que a dor física não impede a realização de atividades. A segunda questiona sobre incômodos físicos em decorrência da infecção, revelando que para 58,5\% não se configura em uma questão negativa. A faceta seguinte avalia a disponibilidade de energia para o diaa-dia, constatando que 60,4\% referiram ter energia suficiente, revelando uma porcentagem maior que outros estudos, sendo $51,1 \%$ em Macaé ${ }^{3}$ e $53 \%$ em Rio das Ostras $^{13}$. Para a quarta faceta, relacionada ao sono, 50,9\% relataram satisfação, apontando uma percepção menos positiva da qualidade de vida, visto que $49,1 \%$ avaliaram negativamente essa faceta.

O domínio nível de independência avalia quatro facetas e foi classificado como intermediário semelhante aos achados na África do $\mathrm{Sul}^{33}$ e Camboja ${ }^{34}$ e, em outros municípios brasileiros, como Macaé ${ }^{3}$, Rio das Ostras ${ }^{13}$, Fortaleza ${ }^{12}$ e Mossoró ${ }^{22}$; já os dados encontrados na Nigéria ${ }^{24}$ e em São José ${ }^{31}$ apresentaram avaliação superior. A primeira faceta questiona a necessidade de tratamento médico e apenas 33,3\% avaliaram positivamente, semelhante aos dados do interior do estado do Rio de Janeiro ${ }^{3,13}$. A faceta que avalia o desempenho nas atividades diárias foi positiva para 70,4\% dos participantes; a faceta que avalia a capacidade para o trabalho, foi positiva para $70,2 \%$ e a capacidade de locomoção foi bem avaliada por $82,2 \%$. 
O domínio meio ambiente apresenta oito facetas relacionadas às condições de vida. Esse domínio apresentou a menor média, embora ainda classificada como intermediária, reafirmando os achados de diversos estudos 3,13,24,31,32,34. A primeira faceta questiona o quão seguro os participantes se sentem na vida diária, que foi respondida positivamente por 60,1\%. A seguinte avalia o quão saudável é o ambiente físico, relacionado ao clima, poluição, entre outros, constatando ser saudável para $47,7 \%$ das pessoas entrevistadas. A terceira faceta questiona sobre a disponibilidade de dinheiro para satisfazer as necessidades, que conforme os achados da variável renda, apontou que para $76,5 \%$ das pessoas o dinheiro é insuficiente, corroborando os achados negativos para $89,3 \%$ dos participantes em Macaé 3 e $87 \%$ em Rio das Ostras $^{13}$. Relacionado à disponibilidade de informações, 55,6\% responderam positivamente a essa quarta faceta. A quinta faceta avaliou a oportunidade para realizar atividades de lazer, que foi positiva para $48,4 \%$ dos participantes. Em relação ao local de moradia, 65,7\% responderam estar satisfeitos ou muito satisfeitos, bem como $63,3 \%$ relataram satisfação com o acesso aos serviços de saúde. Todavia, a faceta sobre a satisfação com o transporte, foi avaliada negativamente por $63 \%$ das pessoas.

\section{CONCLUSÃo}

A qualidade de vida foi positivamente avaliada pelas pessoas vivendo com HIV, principalmente nos domínios psicológico; espiritualidade, religião e crenças pessoais e relações sociais. O destaque da avaliação positiva em dimensões psicossociais revela a importância das relações interpessoais e da rede de apoio social para a garantia de níveis melhores de qualidade de vida, especialmente em uma condição de saúde crônica, que envolve estigma, preconceito e exclusão como grandes desafios a serem enfrentados.

Reconhece-se como limitação deste estudo a sua realização com amostra de conveniência, que restringe a possibilidade de generalização, porém, ressalta-se que a mesma foi composta por pessoas vivendo com HIV, atendidas em serviços públicos de saúde, em cidades com características sociodemográficas diferentes, o que garantiu a variabilidade social, econômica e de nível de escolaridade. Em suma, as características socioeconômicas e clínicas da amostra são semelhantes às observadas em adultos vivendo com HIV no Brasil e em outros países, conforme demonstrado nos resultados: maioria masculina, em atividade laboral, dividida entre baixa e média renda e em uso da TARV.

\section{REFERÊNCIAS}

1. Unaids. Guia de terminologia do UNAIDS. Genebra (Swi): UNAIDS; 2017.

2. Ministério da Saúde (Br). Secretaria de Vigilância em Saúde. Departamento de DST, Aids e Hepatites Virais. Boletim Epidemiológico Aids e DST. Brasília (DF): Secretaria de Vigilância em Saúde; 2015.

3. Costa TL. Representações sociais do HIV/Aids e da qualidade de vida: um estudo entre pessoas que vivem com o agravo em contexto de interiorização [tese de doutorado]. Rio de Janeiro: Universidade do Estado do Rio de Janeiro; 2012.

4. Oliveira DC. Qualidade de vida e construções simbólicas de pessoas que vivem com HIV/aids no Rio de Janeiro. Projeto Edital Universal 2013 do CNPq; 2013.

5. Oliveira DC, Spindola T, Gomes, AMT, Marques SC, Formozo GA, Campos LA. Construções simbólicas do tratamento de pessoas que vivem com o HIV/AIDS entre enfermeiros. Rev. enferm. UERJ [Internet] 2015 [cited 2018 Sep 18]; 23(5):596-602. DOI: https://doi.org/10.12957/reuerj.2015.13191

6. Seidl EMF, Zannon CMLC, Tróccoli BT. Persons living with HIV/AIDS: coping, social support and quality of life. Psicol. Reflex. Crit. [Internet]. 2005 [cited 2018 Sep 18]; 18(2): 188-95. DOI: http://dx.doi.org/10.1590/S0102-79722005000200006

7. Alencar TMD, Nemes MIB, Velloso MA. Transformações da "aids aguda" para a "aids crônica": percepção corporal e intervenções cirúrgicas entre pessoas vivendo com HIV e Aids. Ciênc. saúde coletiva (Online). 2008 [cited 2018 Sep 18]; 13(6):1841-9. DOI: http://dx.doi.org/10.1590/S0104-42302009000200028

8. Organização Mundial da Saúde. Departamento de Saúde Mental e Dependência Química. Instrumento WHOQOL-HIV: sintaxe. Genebra (Swi): OMS; 2002.

9. Fleck MPA, Leal OF, Louzada S, Xavier M, Chachamovich E, Vieira G, Santos L, Pinzon V. Development of the Portuguese version of the OMS evaluation instrument of quality of life. Rev. Bras. Psiquiatr. [Internet]. 1999 [cited 2018 Sep 18]; 21(1):19-28. DOI: http://dx.doi.org/10.1590/S1516-44461999000100006

10. Santos ECM, França Junior I, Lopes F. Quality of life of people living with HIV/AIDS in São Paulo, Brazil. Rev. saúde pública (Online). 2007 [cited 2018 Sep 18]; 41(supl. 2):64-71. http://dx.doi.org/10.1590/S0034-89102007000900011

11. Ministério da Saúde (Br). Secretaria de Vigilância e Departamento de Vigilância, Prevenção e Controle das Infeç̧ões Sexualmente Transmissíveis, do HIV/Aids e das Hepatites Virais. Boletim Epidemiológico Aids e DST. Brasília (DF): Secretaria de Vigilância em Saúde; 2014.

12. Cunha GH, Fiuza MLT, Gir E, Aquino OS, Pinheiro AKB, Galvão MTG. Quality of life of men with AIDS and the model of social determinants of health. Rev. latinoam. enferm. (Online). 2015 [cited 2018 Sep 18];; 23(2):183-91. DOI: http://dx.doi.org/10.1590/0104-1169.0120.2541

13. Hipolito RL. Qualidade de vida das pessoas que vivem com o HIV/Aids no município de Rio das Ostras [tese de doutorado]. Rio de Janeiro: Universidade do Estado do Rio de Janeiro; 2015. 
14. Oliveira FBM, Moura MEB, Araújo TME, Andrade EMLR. Quality of life and associated factors in people living with HIV/AIDS Acta Paul. Enferm. (Online). 2015 [cited 2018 Sep 18]; 28(6):510-6. DOI: http://dx.doi.org/10.1590/1982-0194201500086

15. Silva ACO. Qualidade de vida de pessoas vivendo com HIV/aids e sua associação com aspectos sócio-demográficos, clínicos, psicoemocionais e adesão ao tratamento [tese de doutorado]. Ribeirão Preto; Universidade de São Paulo, 2013.

16. Senado Federal (Br). Lei de diretrizes e bases da educação nacional. Brasília (DF): Coordenação de Edições Técnicas; 2017.

17. Leadebal ODCP, Medeiros LB, Lins RSM, Chaves RB, Monroe AA, Nogueira JA. Cuidado às pessoas vivendo com AIDS: enfoque nas ações de educação em saúde. Rev. enferm. UERJ [Internet] 2017 [cited 2018 Sep 18]; 25 :e9524. DOI: https://doi.org/10.12957/reuerj.2017.9524

18. Castrighini CC, Reis RK, Neves LAS, Galvão MTG, Gir E. Prevalence and epidemiological aspects of HIV/tuberculosis coinfection. Rev. enferm. UERJ [Internet]. 2017 [cited 2018 Sep 18]; 25:e17432. DOI: http://dx.doi.org/10.12957/reuerj.2017.17432

19. Passos SMK, Souza LDM. An evaluation of quality of life and its determinants among people living with HIV/AIDS from Southern Brazil. Cad. Saúde Pública (Online). 2015 [cited 2018 Sep 18]; 31(4):800-14. http://dx.doi.org/10.1590/0102-311X00000514

20. Cardoso Junior JC. O Brasil na encruzilhada: apontamentos para uma reforma do Estado de natureza republicana, democrática e desenvolvimentista. Cadernos do Desenvolvimento [Internet]. 2017 [cited 2018 Sep 18]; 12(20):99-133. Available from: http://www.cadernosdodesenvolvimento.org.br/ojs-2.4.8/index.php/cdes/article/view/31/pdf

21. Reis RK, Santos CB, Dantas RAS, Gir E. Quality of life, sociodemografic factors and sexuality of people living with HIV/AIDS.. Texto contexto enferm [Internet]. 2011 [cited 2018 Sep 18]; 20(3):565-75. DOI: http://dx.doi.org/10.1590/S010407072011000300019

22. Parker R. Unintended consequences: evaluating the impact of HIV and AIDS on sexuality research and policy debates. Cad. Saúde Pública (Online). 2009 [cited 2018 Sep 18]; 25(supl. 2):S251-8. DOI: http://dx.doi.org/10.1590/S0102$311 \times 2009001400007$

23. Coutinho RZ, Miranda-Ribeiro P. Religion, religiosity and sexual initiation during adolescence: lessons from a systematic literature review of a half-century of research. Rev. bras. estud. popul [Internet]. 2014 [cited 2018 Sep 18]; 31(2):333-65. DOI: http://dx.doi.org/10.1590/S0102-30982014000200006

24. Akinboro AO, Akinyemi SO, Olaitan PB, Raji AA, Popoola AA, Awoyemi OR, Ayodele OE. Quality of life of Nigerians living with human immunodeficiency virus. Pan African Medical Journal [Internet]. 2014 [cited 2018 Sep 18]; 18(234). DOI: http://dx.doi.org/10.11604/pamj.2014.18.234.2816

25. Bakiono F, Ouédraogo L, Sanou M, Samadoulougou S, Guigemdé PWL, Kirakoya-Samadoulougou F, Robert A. Quality of life in people living with HIV: a cross-sectional study in Ouagadougou, Burkina Faso. Springerplus [Internet].2014 [cited 2018 Sep 18]; 3(372). DOI: http://dx.doi.org/10.1186/2193-1801-3-372

26. Secretaria Municipal de Saúde do Rio de Janeiro. Boletim epidemiológico DST/aids. Rio de Janeiro (RJ): Secretaria Municipal de Saúde; 2013.

27. Unaids. 90-90-90 uma meta ambiciosa de tratamento para contribuir para o fim da epidemia de AIDS. Genebra (Swi): UNAIDS; 2015.

28. Gomes AMT, Silva EMP, Oliveira DC. Social representations of AIDS and their quotidian interfaces for people living with HIV. Rev. latinoam. enferm. (Online). 2011 [cited 2018 Sep 18]; 19(3): [8 telas]. DOI: http://dx.doi.org/10.1590/S010411692011000300006

29. Hipolito RL, Oliveira DC, Gomes AMT, Costa TL. Social representations of quality of life in HIV/AIDS: the role of time since diagnosis. Rev. enferm. UERJ [Internet]. 2014 [cited 2018 Sep 18]; 22(6):753-9. DOI: https://doi.org/10.12957/reuerj.2014.12840

30. Souto BGA. O HIV, seu portador e o tratamento anti-retroviral: implicações existenciais. São Carlos: EdUFSCar; 2008.

31. Silva J, Bunn K, Bertoni RF, Neves OA, Traebert J. Quality of life of people living with HIV. AIDS Care [Internet]. 2013 [cited 2018 Sep 18]; 25(1):71-6. DOI: DOI: http://dx.doi.org/10.1080/09540121.2012.686594

32. Silva JG, Morgan DA, Melo FCM, Santos IK, Azevedo KPM, Medeiros HJ, Knackfuss MI. Level of pain and quality of life of people living with HIV/AIDS pain and quality of life in HIV/AIDS. AIDS Care [Internet]. 2017 [cited 2018 Sep 18]; 29(8):1041-8. DOI: https://doi.org/10.1080/09540121.2016.1274013

33. Cronje JH, Williams M, Steenkamp L, Venter D, Elkonin D. The quality of life of HIV-infected South African university students: Experiences with the WHOQOL-HIV-Bref. AIDS Care [Internet]. 2017 [cited 2018 Sep 18]; 29(5):632-5. http://dx.doi.org/10.1080/09540121.2016.1234688

34. Yang $Y$, Thai S, Choi J. An evaluation of quality of life among Cambodian adults living with HIV/AIDS and using antiretroviral therapy: a short report. AIDS Care [Internet]. 2016 [cited 2018 Sep 18]; 28(12):1546-50. DOI: http://dx.doi.org/10.1080/09540121.2016.1192100 